\title{
Temperature-dependent high-frequency performance of deep submicron AIGaN/GaN HEMTs
}

\author{
R. Cuerdo , Y. Pei , F. Recht ${ }^{1}$, N. Fichtenbaum , S. Keller , S. P. Denbaars , F. Calle , and U. K. Mishra \\ Electrical and Computer Engineering Department, University of California Santa Barbara, 93106-9560, Santa Barbara, CA, USA \\ Instituto de Sistemas Optoelectrónicos y Microtecnología and Dpto. de Ingeniería Electrónica, E.T.S.I. Telecomunicación, \\ Universidad Politécnica de Madrid, Ciudad Universitaria, 28040 Madrid, Spain
}

A study of the low temperature DC and RF performance of deep submicron $\mathrm{AlGaN} / \mathrm{GaN}$ high electron mobility transistors (HEMTs) is reported. From $300 \mathrm{~K}$ to $100 \mathrm{~K}$ both extrinsic transconductance and drain current increase by $\sim 30 \%$, mainly due to the lowering of the optical phonon scattering that allows higher electron mobility. Source and drain resistances improve too, which contributes to the $15-20 \%$ increase of $f_{t}$ and $f_{\max }$. The low temperature small signal model has

1 Introduction $\mathrm{AlGaN} / \mathrm{GaN}$ high electron mobility transistors (HEMTs) are being used in monolithic microwave integrated circuits (MMIC) for power applications at $\mathrm{X}$ band and above for the last few years. They take advantage of some interesting properties of III-nitrides, such as their intense piezoelectric fields, high breakdown field and saturation velocity, and good thermal conductivity, to provide high power at increasing frequencies and harsh environments. To fully understand the HEMT performance from a thermal point of view, a complete characterization of the devices should be done over a wide range of temperatures. For high temperatures (HT), Lee and Webb reported a temperature dependent nonlinear model on a $0.35 \mu \mathrm{m}$ gate length device from $300 \mathrm{~K}$ to $423 \mathrm{~K}$ On the other hand, Nidhi has studied the impact of access resistance on HEMTs with high frequency performance for cryogenic temperatures This work completes the study at low temperatures (LT) by means of extracting the small signal model from $300 \mathrm{~K}$ down to $100 \mathrm{~K}$ at every $50 \mathrm{~K}$. also been extracted accurately at every $50 \mathrm{~K}$. Inductances and capacitances remain constant in the range of temperatures measured. The intrinsic transconductance can be also considered temperature independent, but the output conductance decreases from $300 \mathrm{~K}$ to $100 \mathrm{~K}$ indicating a better confinement of the 2DEG. The HEMT performance obtained at $100 \mathrm{~K}$ can be reached at room temperature by reducing the parasitic resistances and improving the GaN buffer isolation.

2 Experimental $\mathrm{Al}_{0.33} \mathrm{Ga}_{0.67} \mathrm{~N} / \mathrm{GaN}$ HEMTs with 20 $\mathrm{nm}$ barriers were grown by metal-organic chemical vapour deposition (MOCVD) on SiC. A $0.6 \mathrm{~nm}$ AlN interlayer between the buffer and the barrier was used to improve the 2DEG transport properties. In order to achieve a nonalloyed low resistance ohmic contact $\left(\mathrm{R}_{\mathrm{c}} \approx 0.2 \Omega \cdot \mathrm{mm}\right), \mathrm{Si}$ ions were implanted into the source and drain regions and then activated by annealing the sample at $\sim 1280{ }^{\circ} \mathrm{C}$ in a MOCVD system with a $\mathrm{N}_{2}$ and $\mathrm{NH}_{3}$ ambient The sample was then passivated by $\mathrm{SiN}_{\mathrm{x}}$ and a sacrificial Ge layer was deposited to decrease parasitic capacitances. Submicron gates were defined by e-beam lithography and dryetched through the $\mathrm{Ge}$ and $\mathrm{SiN}_{\mathrm{x}}$ layers. The gate region was treated by fluorine plasma for 10 minutes to decrease the gate leakage, and the AlGaN layer recessed by chlorine to improve the transconductance. Transistors have a gate length $\sim 150 \mathrm{~nm}$ with gate-source spacing $\left(\mathrm{L}_{\mathrm{GS}}\right)$ of $0.3 \mu \mathrm{m}$ and drain-gate spacing $\left(\mathrm{L}_{\mathrm{DG}}\right)$ of $1.1 \mu \mathrm{m}$. Gate widths are of $100 \mu \mathrm{m}$ or $150 \mu \mathrm{m}$. The sheet carrier density $\left(\mathrm{n}_{\mathrm{s}}\right)$ and Hall mobility $\left(\mu_{\mathrm{H}}\right)$ were $1.1 \cdot 10^{13} \mathrm{~cm}^{-2}$ and $2000 \mathrm{~cm}^{2} / \mathrm{V} \cdot \mathrm{s}$. 
S-parameter measurements from $300 \mathrm{~K}$ to $100 \mathrm{~K}$ were carried out using a cryogenic high frequency probe station, composed of a liquid nitrogen cooled wafer stage and a heater to control the temperature. The station was connected to an Agilent PNA E8361A network analyzer for the RF measurements and to an Agilent 4155B parameter analyzer for the DC characterization. For the on wafer RF measurements from $1 \mathrm{GHz}$ up to $40 \mathrm{GHz}$, the electrical reference plane was established by an "off-wafer" LRRM calibration at each temperature.

3 Results and discussion Both drain cut-off current ( $\left.\mathrm{I}_{\mathrm{DSS}}\right)$ and extrinsic transconductance $\left(\mathrm{g}_{\mathrm{m}, \mathrm{ext}}\right)$ increase around $30 \%$ from $300 \mathrm{~K}$ to $100 \mathrm{~K}$ as shown in Fig. 1, up to maximum values of $0.99 \mathrm{~A} / \mathrm{mm}$ and $0.62 \mathrm{~S} / \mathrm{mm}$, respectively. This behaviour is related with the higher electron mobility in the $2 \mathrm{DEG}$ as devices are cooling, due to the reduction of the polar optical phonon scattering. This mechanism is dominant at $300 \mathrm{~K}$, but becomes negligible as temperature decreases. At $100 \mathrm{~K}$ other types of scattering such as the alloy disorder are predominant with a temperature independent behaviour. Thus, the improvement in the HEMT performance saturates for $\mathrm{T}<150 \mathrm{~K}$.

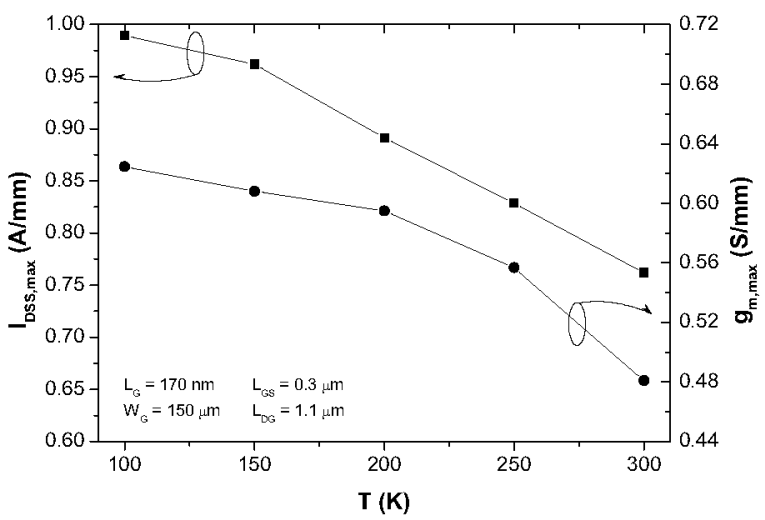

Figure 1 Maximum of drain current at $\mathrm{V}_{\mathrm{GS}}=0 \mathrm{~V}$ and extrinsic transconductance at $\mathrm{V}_{\mathrm{DS}}=8 \mathrm{~V}$ from $300 \mathrm{~K}$ to $100 \mathrm{~K}$.

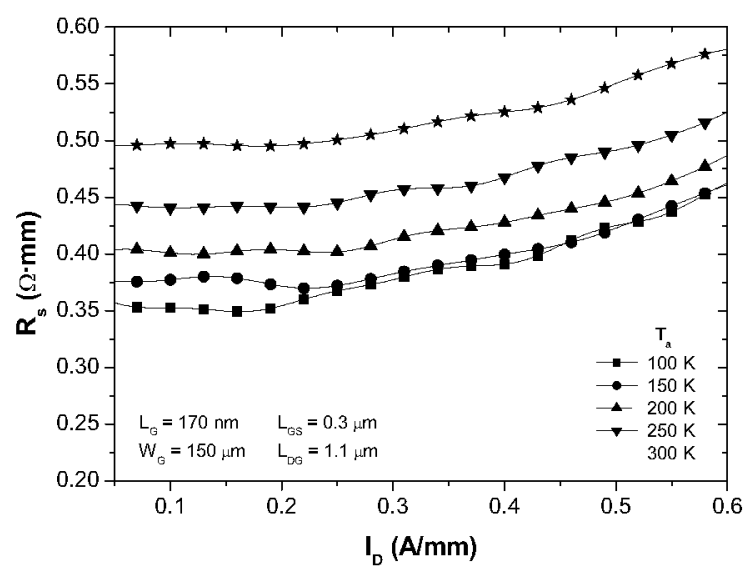

Figure $2 R_{S}$ for different drain currents. At each $T$, the minimum of $R_{S}$ is obtained in the low field region for $I_{D}<0.2 \mathrm{~A} / \mathrm{mm}$.
The source resistance $\left(R_{S}\right)$ has been extracted from specific DC measurements For transistors with $\mathrm{L}_{\mathrm{GS}}=$ $0.3 \mu \mathrm{m}$ and $\mathrm{L}_{\mathrm{DG}}=1.1 \mu \mathrm{m}, \mathrm{R}_{\mathrm{S}}$ decreases from $0.49 \Omega \cdot \mathrm{mm}$ at $300 \mathrm{~K}$ to $0.35 \Omega \cdot \mathrm{mm}$ at $100 \mathrm{~K}$ as is shown in Fig. 2 . Drain resistance $\left(R_{D}\right)$ can be also calculated as the difference between the on-resistance $\left(R_{o n}\right)$ and $R_{S}$, extracting $R_{\text {on }}$ from the slope of the lineal region in $I_{D}$ vs $V_{D S}$ curves at low $\mathrm{V}_{\mathrm{DS}}$. For the same devices, $\mathrm{R}_{\mathrm{D}}$ varies from $1.15 \Omega \cdot \mathrm{mm}$ at $300 \mathrm{~K}$ to $0.65 \Omega \cdot \mathrm{mm}$ at $100 \mathrm{~K}$.

The measurements of the S-parameters allow the extraction of the current-gain $\left(\mathrm{f}_{\mathrm{T}}\right)$ and power-gain $\left(\mathrm{f}_{\max }\right)$ cutoff frequencies and the equivalent circuit parameters (ECPs). The $f_{T}$ and $f_{\max }$ are estimated from $\left|h_{21}\right|^{2}$ and the unilateral power gain (U), respectively, assuming a 20 $\mathrm{dB} /$ decade linear decrease. As shown in Fig. 3, $\mathbf{f}_{\mathrm{T}}$ increases $\sim 16 \%$ from $300 \mathrm{~K}$ to $150 \mathrm{~K}$, and stabilizes down to $100 \mathrm{~K}$. The maximum of $f_{T}$ occurs approximately at the same $I_{D}$ for all temperatures under study The $f_{\max }$ follows a similar evolution than $\mathrm{f}_{\mathrm{T}}$, increasing $\sim 21 \%$ from $129 \mathrm{GHz}$ at $300 \mathrm{~K}$ to $156 \mathrm{GHz}$ at $100 \mathrm{~K}$. Both $\mathrm{f}_{\mathrm{T}}$ and $\mathrm{f}_{\max }$ increases are related with the improvement of the parasitic resistances $\left(\mathrm{R}_{\mathrm{S}}\right.$ and $\left.\mathrm{R}_{\mathrm{D}}\right)$ as devices are cooled

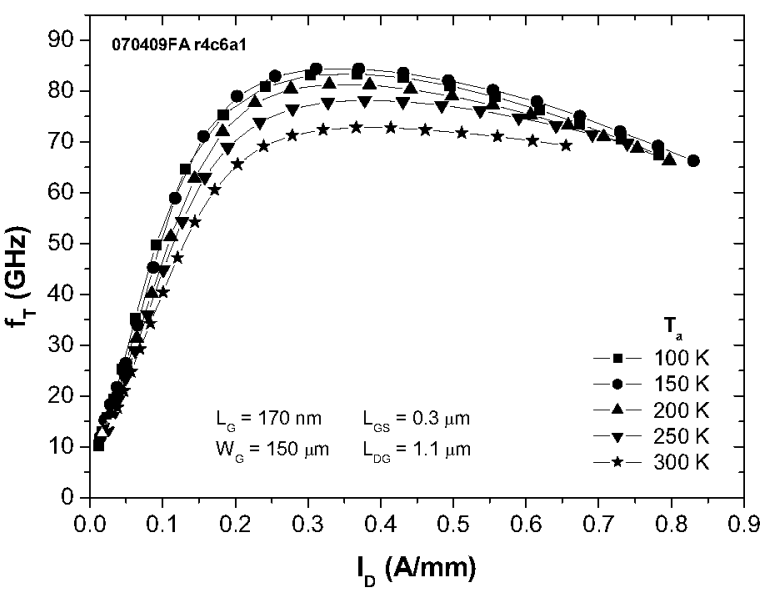

Figure 3 Evolution of $f_{T}$ for different drain currents. The peak of $f_{T}$ occurs at the same approximate $I_{D}(0.3$ to $0.4 \mathrm{~A} / \mathrm{mm})$ for all temperatures under study.

The small signal model has been extracted and simulated at different bias by means of ADS software. The equivalent circuit used was proposed by Dambrine et al.

The extrinsic resistances $R_{s}$ and $R_{d}$ have been previously calculated, and $R_{g}$ is set as $1 / 3$ of the gate metal strip resistance. From pinched HEMT measurements, extrinsic capacitances $\mathrm{C}_{\mathrm{pg}}$ and $\mathrm{C}_{\mathrm{pd}}$ are obtained. The extrinsic inductances $L_{s}, L_{g}$ and $L_{d}$ are calculated from cold measurements $\left(\mathrm{V}_{\mathrm{DS}}=0 \mathrm{~V}\right.$ and $\left.\mathrm{V}_{\mathrm{GS}} \approx 2 \mathrm{~V}\right)$. Next, the intrinsic circuit parameters are deduced following The equivalent circuit is simulated and fitted to achieve a good agreement between model and measured characteristics, especially for frequencies between 1 to $20 \mathrm{GHz}$ (see Fig. 4). Inductances and capacitances for the extrinsic circuit are supposed constant with temperature. Regarding the 


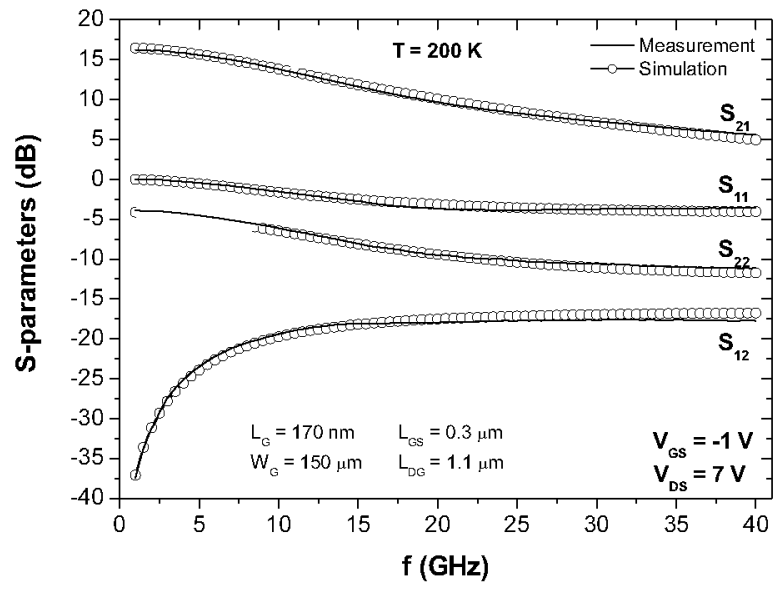

Figure 4 Comparison between the S-parameters measured with the network analyzer and simulated by ADS from the small signal equivalent circuit.

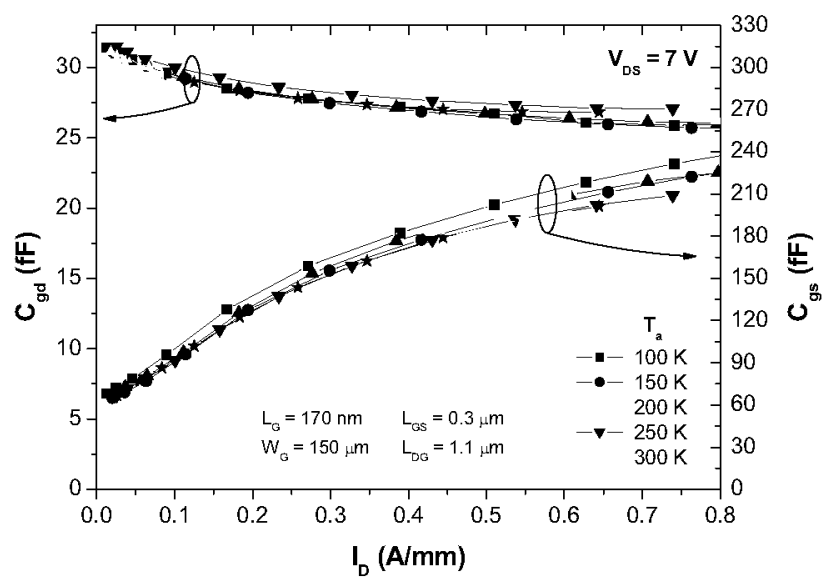

Figure $5 \mathrm{C}_{\mathrm{gd}}$ and $\mathrm{C}_{\mathrm{gs}}$ temperature evolution for $\mathrm{V}_{\mathrm{DS}}=7 \mathrm{~V}$ with $\mathrm{V}_{\mathrm{GS}}$ bias between $-2 \mathrm{~V}$ and $0 \mathrm{~V}$.

intrinsic circuit, all parameters have been calculated for different $\mathrm{I}_{\mathrm{D}}$ (keeping $\mathrm{V}_{\mathrm{DS}}$ constant). $\mathrm{C}_{\mathrm{gs}}$ and $\mathrm{C}_{\mathrm{gd}}$ can be considered temperature independent, since their variations are lower than 7\%, as shown in Fig. 5.

In addition, the intrinsic transconductance $\left(\mathrm{g}_{\mathrm{m}, \mathrm{int}}\right)$ is almost constant with $T$, so $g_{m, e x t}$ mainly depends on $R_{S}$ as:

$$
g_{m, \text { ext }}=\frac{g_{m, \text { int }}}{1+R_{S} \cdot g_{m, \text { int }}}
$$

The output conductance $\left(\mathrm{g}_{\mathrm{ds}}\right)$ decreases $\sim 30 \%$ as devices are cooled from $300 \mathrm{~K}$ to $100 \mathrm{~K}$, likely due to a better confinement of the electrons in the heterostructure (see Fig. 6). In fact, simulations of the electron density across the transistor show that redistribution of the electrons in the $\mathrm{AlGaN} / \mathrm{GaN}$ interface when the temperature is lowered (inset to Fig. 6), suggesting an improvement of the isolation of the GaN buffer.

Other intrinsic parameters $\left(C_{d s}, R_{i}\right.$ or $\left.\tau\right)$ do not show a clear changes at low $\mathrm{T}$.

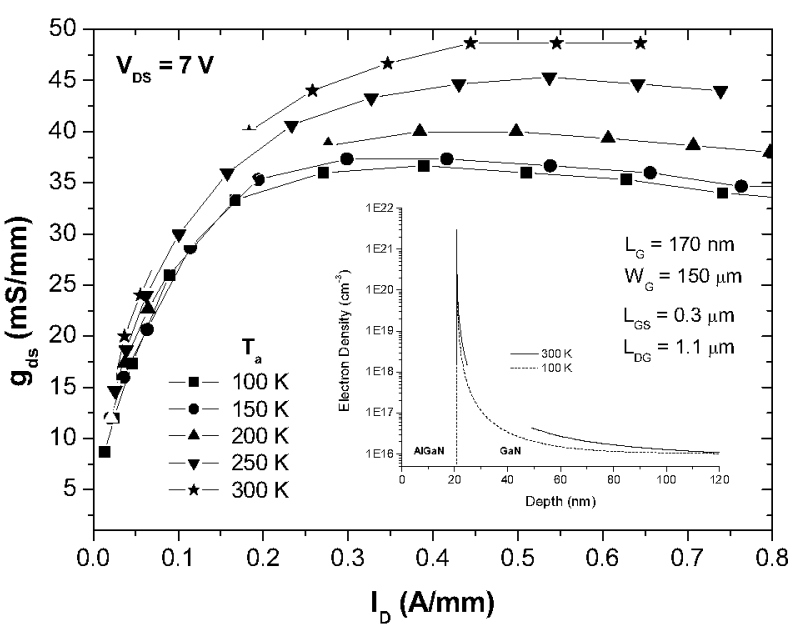

Figure 6 Output conductance evolution from $300 \mathrm{~K}$ to $100 \mathrm{~K}$ for different drain currents. In the inset, electron density simulations show that low temperatures improve the $2 \mathrm{DEG}$ confinement.

4 Conclusion Low temperature measurements allow the determination of the dominant limiting factors of the HEMT performance. From $300 \mathrm{~K}$ down to $100 \mathrm{~K}, \mathrm{R}_{\mathrm{S}}$ and $R_{D}$ experience a clear decrease above $40 \%$, which improves not only the DC performance ( $I_{D}$ and $\left.g_{m, c x t}\right)$ but also $\mathrm{f}_{\mathrm{T}}$ and $\mathrm{f}_{\max }$.

Every $50 \mathrm{~K}$ the ECPs of several transistors have been extracted in an accurate way. Apart from the parasitic resistances behaviour, $g_{d s}$ shows a significant reduction at low temperatures. This fact suggests a better buffer isolation, as also confirmed by the decrease of leakage currents and by the better 2DEG confinement deduced from simulations. Other parameters $\left(\mathrm{g}_{\mathrm{m}, \mathrm{int}}, \tau, \mathrm{C}_{\mathrm{gd}}, \mathrm{C}_{\mathrm{gs}}\right)$ from the small signal model seem to be rather constant with temperature for this type of deep gate transistors.

\section{References}

U. K. Mishra, P. Parikh, and Y. F. Wu, Proc. IEEE 90, 1022 (2002).

Y. F. Wu, A. Saxler, M. Moore, R. P. Smith, S. Sheppard, P. M. Chavarkar, T. Wisleder, U. K. Mishra, and P. Parikh, IEEE Electron Dev. Lett. 25, 117 (2004).

J. Lee and K. J. Webb, IEEE Trans. Microw. Theory Tech. 52, 2 (2004)

Nidhi, T. Palacios, A. Chakraborty, S. Keller, and U. K. Mishra, IEEE Electron Dev. Lett. 27, 877 (2006).

R. Recht, L. McCarthy, L. Shen, C. Poblenz, A. Corrion, J. S. Speck, and U. K. Mishra, 65th DRC digest, 37 (2007).

Y. Pei, L. Shen, T. Palacios, N. A. Fichtenbaum, S. Keller, S. P. Denbaars, and U. K. Mishra, Jpn. J. Appl. Phys. 46, 842 (2007). 
G. Dambrine, A. Cappy, F. Heliodore, and E. Playez, IEEE Trans. Microwave Theory and Tech. 36, 1151 (1988).

R. Anholt, Electrical and Thermal Characterization of MESFETs, HEMTs, and HBTs (Artech, Boston, 1995), chap. 3.

J. Michael Golio, Microwave MESFETs \& HEMTs (Artech, Boston, 1991), chap. 4. 